九州大学学術情報リポジトリ

Kyushu University Institutional Repository

\title{
A Note on a Nonparametric Two-Sample Test
}

Ishi i, Goro

Atomic Bomb Casuality Comission

https://doi.org/10.5109/12994

出版情報 : 統計数理研究. 9 (2/3)，pp. 57-60，1960-10. Research Association of Statistical Sciences

バージョン :

権利関係 : 


\title{
A NOTE ON A NONPARAMETRIC TWO-SAMPLE 'TEST
}

By

\author{
Goro ISHII \\ (Received December 5, 1959) \\ (Revised January 31, 1960)
}

\section{Introduction}

Let $X$ and $Y$ be the two random variables which follow the distribution functions of continuous type $F(x)$ and $G(y)$, respectively. The test of the hypothesis $H_{0}: F(x)=G(x)$ is treated here. For this purpose a slightly modified test of David-Okamoto's test [2], [6] will be applied.

Let $x_{i}, i=1,2, \cdots, N$, and $y_{j}, j=1,2, \cdots, M$, be $N$ and $M$ observations of $X$ and $Y$. These $x_{i}$ and $y_{j}$ are mixed and arranged in order of magnitude. Denote these by

$$
z_{1}<z_{2}<\cdots \cdots \cdots \cdots \cdots \cdots+z_{L}
$$

where $L=N+M$. We shall consider here only the case when $L=n \alpha$ where $\alpha$ is a fixed integer. Divide (1) into $n$ classes from the left so that each class contains $\alpha$ members. That is

$$
z_{1}, z_{2}, \cdots, z_{\alpha} ; z_{\alpha+1}, \cdots, z_{2 x} ; \cdots ; \cdots, z_{n \alpha} \text {. }
$$

The number of $x_{i}$ involved in each clase is denoted by $i_{k}, k=1,2, \cdots, n$. Consider a function of $i$

$$
\begin{aligned}
h(i) & =0, \quad \text { when } i \Longleftarrow 0, \\
& =1, \quad \text { when } i=0 .
\end{aligned}
$$

Then

$$
v=\sum_{k=1}^{n} h\left(i_{k}\right)
$$

is the number of classes which contain no $x$ 's. And $u=n-v$ is the number of classes which contain some $x$ 's.

A large value of $v$ suggests that the hypothesis should be rejected.

Therefore we use the $v$ as a test function and call this test $v$-test.

In many occations, a test of goodness of fit can be extended to a two sample test, Darling [1]. For example, Kolmogorov's test of fit by $D_{n}=$ $\sup \left|F(x)-F_{n}(x)\right|$ has a corresponding two sample test by $D_{n, m}=\sup \mid F_{n}(x)$ $-G_{m}(x) \mid$ while $\omega^{2}$ test of fit by $\omega_{n}^{2}=n \int_{-\infty}^{\infty}\left(F(x)-F_{n}(x)\right)^{2} d F(x)$ has a correspon- 
ding two sample test by $\omega_{n, m}^{2}=\frac{m n}{m+n} \int_{-\infty}^{\infty}\left(F_{n}(x)-G_{m}(x)\right)^{2} d^{n F_{n}+m G_{m}} \frac{n+m}{n+m}$.

David [2] proposed a test of fit by the following procedure. Let $x_{i}$, $i=1,2, \cdots, N$, be $N$ independent observations of $X$. Since $F(x)$ is continuous, there are real numbers $\left\{a_{i}\right\}, i=0,1,2, \cdots, n-1$ such that $F\left(a_{i+1}\right)-F\left(a_{i}\right)=$ $1 / n$ where $a_{0}=-\infty$ and $a_{n}=+\infty$. Let $v_{0}$ be the number of intervals $\left(a_{i-1}, a_{i}\right)$ which contain no $x$ 's. If $v_{0}$ is too large, the hypothesis that the distribution of $X$ is $F(x)$ will be rejected. The detailed character of this test was treated by Okamoto [6], Weiss [7], Kitabatake [5], and the application of the test to life testing was treated by Ishii [4]. It is clear that the $v$-test above mentioned is a two sample test corresponding to the test of fit by David-Okamoto.

\section{Distribution of $v$}

Let us consider the behavior of $x$ 's in series (2). The probability that $N x$ 's are contained in at most $k$ classes is

$$
\left(\begin{array}{c}
k \alpha \\
N
\end{array}\right) /\left(\begin{array}{l}
L \\
N
\end{array}\right)
$$

This expression has a meaning only when $k \alpha \geqq N$. When $k \alpha<N$, we let the probability 0 .

Denote by $p_{i}$ the probability that $i$ classes in the above $k$ classes contain all of the $N x$ 's and that each contains at least one of the $x$ 's. Then we have

$$
\left(\begin{array}{c}
k \alpha \\
N
\end{array}\right) /\left(\begin{array}{l}
L \\
N
\end{array}\right)=\sum_{i=1}^{k}\left(\begin{array}{l}
k \\
i
\end{array}\right) p_{i} .
$$

Therefore for every positive integer $\nu$

$$
\begin{aligned}
& \sum_{k=1}^{\nu}(-1)^{\nu-k}\left(\begin{array}{l}
\nu \\
k
\end{array}\right)\left(\begin{array}{l}
k \alpha \\
N
\end{array}\right)\left(\begin{array}{l}
L \\
N
\end{array}\right) \\
& =\sum_{k=1}^{\nu}(-1)^{\nu-k}\left(\begin{array}{l}
\nu \\
k
\end{array}\right) \sum_{i=1}^{k}\left(\begin{array}{l}
k \\
i
\end{array}\right) p_{i} \\
& =\sum_{i=1}^{\nu} p_{i}\left(\begin{array}{l}
\nu \\
i
\end{array}\right) \sum_{k=i}^{\nu}(-1)^{\nu-k}\left(\begin{array}{l}
\nu-i \\
k-i
\end{array}\right)=p_{i} .
\end{aligned}
$$

Thus

$$
P\{u=\nu\}=\left(\begin{array}{l}
n \\
\nu
\end{array}\right) p_{\nu}=\left(\begin{array}{l}
n \\
\nu
\end{array}\right) \sum_{k=1}^{\nu}(-1)^{\nu-k}\left(\begin{array}{l}
\nu \\
k
\end{array}\right)\left(\begin{array}{l}
k \alpha \\
N
\end{array}\right) /\left(\begin{array}{l}
L \\
N
\end{array}\right) .
$$

Then

$$
P\{v=\nu\}=\left(\begin{array}{l}
n \\
\nu
\end{array}\right) \sum_{k=1}^{n-\nu}(-1)^{n-\nu-k}\left(\begin{array}{c}
n-\nu \\
k
\end{array}\right)\left(\begin{array}{c}
k \alpha \\
N
\end{array}\right) /\left(\begin{array}{l}
L \\
N
\end{array}\right) \quad \nu=0,1, \cdots, n-1 .
$$

The $s$-th factorial moment of $v$ is

$$
E\left(v^{(s)}\right)=\frac{((n-s) \alpha)^{(N)} n !}{(n \alpha)^{(v)}(n-s) !}
$$

where

$$
x^{(s)}=x(x-1) \cdots(x-s+1) .
$$


Putting $\quad s=1,2$

$$
\begin{aligned}
& E(v)=n \frac{(L-\alpha)^{(n)}}{L^{(i)}} \\
& E(v(v-1))=n(n-1) \frac{(L-2 \alpha)^{(v)}}{L^{(i)}}
\end{aligned}
$$

In the case when $N$ is fixed and $M$ tends to infinity, the probability (4) tends to $p(v=v)$ in Okamoto [6].

In the case when both $N$ and $M$ tend to infinity, we can assume many conditions on the increasing order of $N, M, \alpha$ and $n$. In the following we shall treat the case that $\alpha$ and $n$ tend to infinity with order $\checkmark \bar{L}$ and $M=n$ $(\alpha-r), N=n r$ where $r$ is fixed. In this case the factorial moment (5) becomes

$$
E\left(v^{(s)}\right) \sim\left(1-\frac{s}{n}\right)^{n r} \frac{n !}{(n-s) !} .
$$

On the asymptotic behavior of $v$ the following theorem is developed. The proof is similar to that of theorem 1 in Okamoto [6] and theorem 1 in Ishii [4]. Therefore, the proof is omitted.

\section{Theorem 1 .}

$v / n$ is asymptotically normally distributed with mean $e^{-r}$ and variance $e^{-2 r}\left(e^{r}-1-r\right) / n$, where $r=N / n=$ const.

\section{Application to life test.}

Suppose that life testing of $X$ and $Y$ start with $N x$ items and $M y$ items and stops when $L_{1}=n_{1} \alpha$ deaths in total occur, where $L_{1}$ is a certain preassigned number. Let $z_{i}, i=1,2, \cdots, L_{1}$, be the ordered observations of $X$ and $Y$. Denote by $N_{1}$ and $M_{1}$ the number of $X$ and $Y$ in $L_{1} z$ 's.

$$
\begin{aligned}
& L_{1}==n_{1} \alpha=N_{1}+M_{1} \\
& L=n \alpha=N+M \\
& L_{1} \leqq L .
\end{aligned}
$$

Divide these $z_{i}$ into $n_{1}$ classes in the same way as in section 1

$$
z_{1}, z_{2}, \cdots, z_{\alpha} ; z_{\alpha+1}, \cdots, z_{2 \alpha} ; \cdots, z_{n 1 \alpha} .
$$

Let $v_{1}$ be the number of classes that contain no $x$ 's in the above $n_{1}$ classes. We shall use the $v_{1}$ as a test function of testing the hypothesis $H_{0}$.

$N_{1}$ follows the hypergeometric distribution

$$
p\left\{N_{1}\right\}=\left(\begin{array}{l}
N \\
N_{1}
\end{array}\right)\left(\begin{array}{l}
M \\
M_{1}
\end{array}\right) /\left(\begin{array}{l}
L \\
L_{1}
\end{array}\right) \text {. }
$$

The probability of $v_{1}$ under the condition that $N_{1}$ is fixed is 


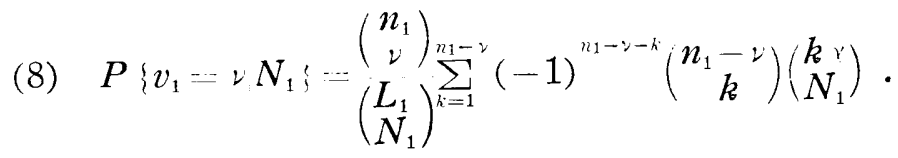
Then

(9) $\boldsymbol{P}\left\{v_{1}=\nu, N_{1}\right\}=P\left\{v_{1}=\nu N_{1}\right\} \cdot P\left\{N_{1}\right\}$

(10) $P\left\{v_{1}=\nu\right\}=\sum_{v_{1}} P\left\{v_{1}=\nu, N_{1}\right\}$.

The $s$-th factorial moment of $v_{1}$ is

$$
E\left(v_{1}^{(s)}\right)=\frac{((n-s) \alpha)^{(n)} n_{1} !}{(n x)^{(N)}\left(n_{1}-s\right) !} .
$$

Putting $\quad s=1,2$

$$
\begin{gathered}
E\left(v_{1}\right)=n_{1} \frac{(L-\alpha)^{(N)}}{L^{(N)}} \\
E\left(v_{1}\left(v_{1}-1\right)\right)=n_{1}\left(n_{1}-1\right) \frac{(L-2 \alpha)^{(N)}}{L^{(N)}}
\end{gathered}
$$

In the case when $N$ and $n_{1} / n$ are fixed and $M$ tends to infinity, the probability (9) tends to $P\left(v_{1}=\nu\right)$ in Ishii [4.]. In the case when both $N$ and $M$ tend to infinity, we shall add a condition that $n_{1} / n=t$ is fixed to the assumptions in section 2 . Under these assumptions we have the following theorem on the asymptotic behavior of $v_{1}$. The proof is also similar to that of theorem 1 in Ishii [4]. Therefore it is omitted.

\section{Theorem 2 .}

$v_{1} / n_{1}$ is asymptotically normally distributed with mean $e^{-r}$ and variance $e^{-2 r}\left(e^{r}-1-t r\right) / n$, where $r=N / n$ and $t=n_{1} / n$ are constants. Atomic Bomb Casualty Comission, Hiroshima

\section{References}

1. D. A. Darling, “The Kolmogorov-Smirnov, Cramer-Von Mises Tests" Ann. Math. Stat. Vol. 28 (1957), pp. 823-838.

2. F. N. DAvid, “Two combinatorial tests of whether a sample has come from a given population" Biometrika Vol. 37 (1950), pp. 97-110.

3. G. Ismin, "Test of fit in life test" Ann. Inst. Stat. Math. Vol. 9(1957-8), pp. 117-125.

4. G. Ishir, "On a non-parametric test in life test" Bull. Math. Stat. Vol. 8 (1959), pp. 73-79.

5. S. KiтаватакF, “A remark on a non-parametric test" Mathematica Japonicae, Vol. 5 (1958), pp. 45-49.

6. М. Окамото, “On a non-parametric test” Osaka Math. J. Vol. 4 (1952), pp 76-85.

7. I. Weiss, "Limiting distribution in some occupancy problem" Ann. Math. Stat. Vol. 29 (1958), pp. $878-884$. 